IMPROVEMENT of the BOOSTER HEAVY ION INJECTION LINE

BOOSTER TECHNICAL NOTE

NO. 192

J. XU

MAY 14, 1991 


\title{
IMPROVEMENT OF THE BOOSTER HEAVY ION INJECTION LINE
}

\author{
J. Xu \\ Accelerator Development Department \\ Brookhaven National Laboratory
}

May 23, 1991

The layout and beam optics of the new design of the heavy ion injection line for the AGS booster have been described in reference 1 by R.C. Gupta et al. In their design, at the booster injection point there is vertical dispersion mismatch and MAD program was used. I have run the same lattice with both MAD and SYNCH programs. Dr. Milutinovic has run it with program PATRIS. The outputs from different programs are really different slightly. The output of MAD program is shown in Table 1, the input data and output of SYNCH are shown in Table 2 and Table 3 respectively. The Twiss parameters and dispersion functions at the booster injection point from different programs are shown in the following table, Table 4,

Table 4

$\begin{array}{lllllllllll} & \beta_{x} & \alpha_{x} & \eta_{x} & \eta_{x}^{\prime} & \mu_{x} & \beta_{y} & \alpha_{y} & \eta_{y} & \eta_{y}^{\prime} & \mu_{y} \\ \text { MAD } & 13.976 & -0.208 & 2.82 & 0.286 & 1.545 & 3.568 & 0.304 & 0.391 & 0.119 & 0.973 \\ \text { SYNCH } & 13.976 & -0.208 & 2.82 & 0.286 & 1.545 & 3.547 & 0.307 & 0.485 & 0.057 & 0.972 \\ \text { PATRIS }^{2} & 12.550 & -0.073 & 2.82 & 0.286 & 1.552 & 3.514 & 0.359 & 0.391 & 0.119 & 0.967\end{array}$

The dispersion functions from MAD coincide with those from PATRIS and the Twiss parameters from MAD coincide with those from SYNCH. The discrepancy is produced due to the tilt of bending magnets. From the above results, one can assume that, the output of MAD is reliablc cven though in its output there is a warning message.

The focusing parameters and the location of the quadrupoles have been tuned so that to get a better match at the booster injection point. In this process, MAD program was used. The output are shown in Table 5.

Next, the emittance dilution due to dispersion mismatch will be estimated. Suppose, the Twiss parameters in $\mathrm{y}$-direction at the booster injection point are $\alpha, \beta, \gamma, \eta_{y}$ and $\eta_{y}^{\prime}$, the dispersion function of the injection line at the injection point are $\eta_{y \ell}, \eta_{y \ell}^{\prime}$ and momentum error is $\frac{\Delta p}{p}$, then the emittance dilution factor $F$ is expressed as follows ${ }^{3}$

$$
F=\left[1+\sqrt{\frac{\epsilon_{p}}{\epsilon_{o}}}\right]^{2}
$$

where $\epsilon_{o}$ is the emittance of the injected beam and $\epsilon_{p}$ can be expressed as follows

$$
\epsilon_{p}=\left(\frac{\Delta p}{p}\right)^{2}\left[\gamma\left(\eta_{y \ell}-\eta_{y}\right)^{2}+2 \alpha\left(\eta_{y \ell}-\eta_{y}\right)\left(\eta_{y \ell}^{\prime}-\eta_{y}^{\prime}\right)+\beta\left(\eta_{y \ell}^{\prime}-\eta_{y}^{\prime}\right)^{2}\right]
$$


For the original injection line lattice, the emittance dilution factor due to vertical dispersion mismatch will be:

$$
F=\left[1+\frac{\Delta p}{p}\left(\frac{0.126}{\epsilon_{o}}\right)^{1 / 2}\right]^{2}
$$

From reference $4 \epsilon_{o} \approx 1 \mathrm{~mm} \cdot \mathrm{mrad}$, then

$$
F=\left[1+0.35 \frac{\Delta p}{p}\right]^{2}
$$

where $\frac{\Delta p}{p}$ is expressed in $10^{-3}$. The emittance dilution factors $F$ corresponding to various $\frac{\Delta p}{p}$ are shown in the following table,

$\begin{array}{llll}\mathrm{F} & 1.83 & 1.38 & 1.14 \\ \frac{\Delta p}{p}\left(10^{-3}\right) & 1 & 0.5 & 0.2\end{array}$

For the improved lattice, $F$ due to $\eta_{y}^{\prime}$ mismatch can be expressed as follows

$$
F=\left[1+5.7 \times 10^{-3} \frac{\Delta p}{p}\right]^{2} \text {. }
$$

$F$ due to $\beta_{y}, \alpha_{y}$ small mismatch is 1.007 . Both $F$ 's equal 1 approximately. The emittance dilution is negligibly small. One thing should be pointed out is that the maximum $\beta_{y}$ in this lattice is approximately 100 meters, but the beam size remains well within the $4^{\prime \prime}$ aperture of the quadrupoles. Another possibility to improve this line is to tune the upstream line focusing parameters or to tune the whole line from Tandem to booster injection point.

\section{Acknowledgments}

I am thankful to Dr. A.G. Ruggiero for valuable discussion and Dr. S. Tepikian and J. Milutinovic for their help in running SYNCH program.

\section{References}

1. R.C. Gupta, R. Damm, Y.Y. Lee and W.T. Weng, $H^{-}$and Heavy Ion Injection Lines for the Booster, Booster Technical Note No. 90, Sept. 1987.

2. J. Milutinovic, private communication, 1991.

3. J. Xu, J. Claus and A.G. Ruggiero, A Feedback Device to Damp the Coherent Oscillations from Injection Errors in RHIC, AD/RHIC Technical Note No 74, 1990.

4. Booster Design Manual, p. 4-16. 


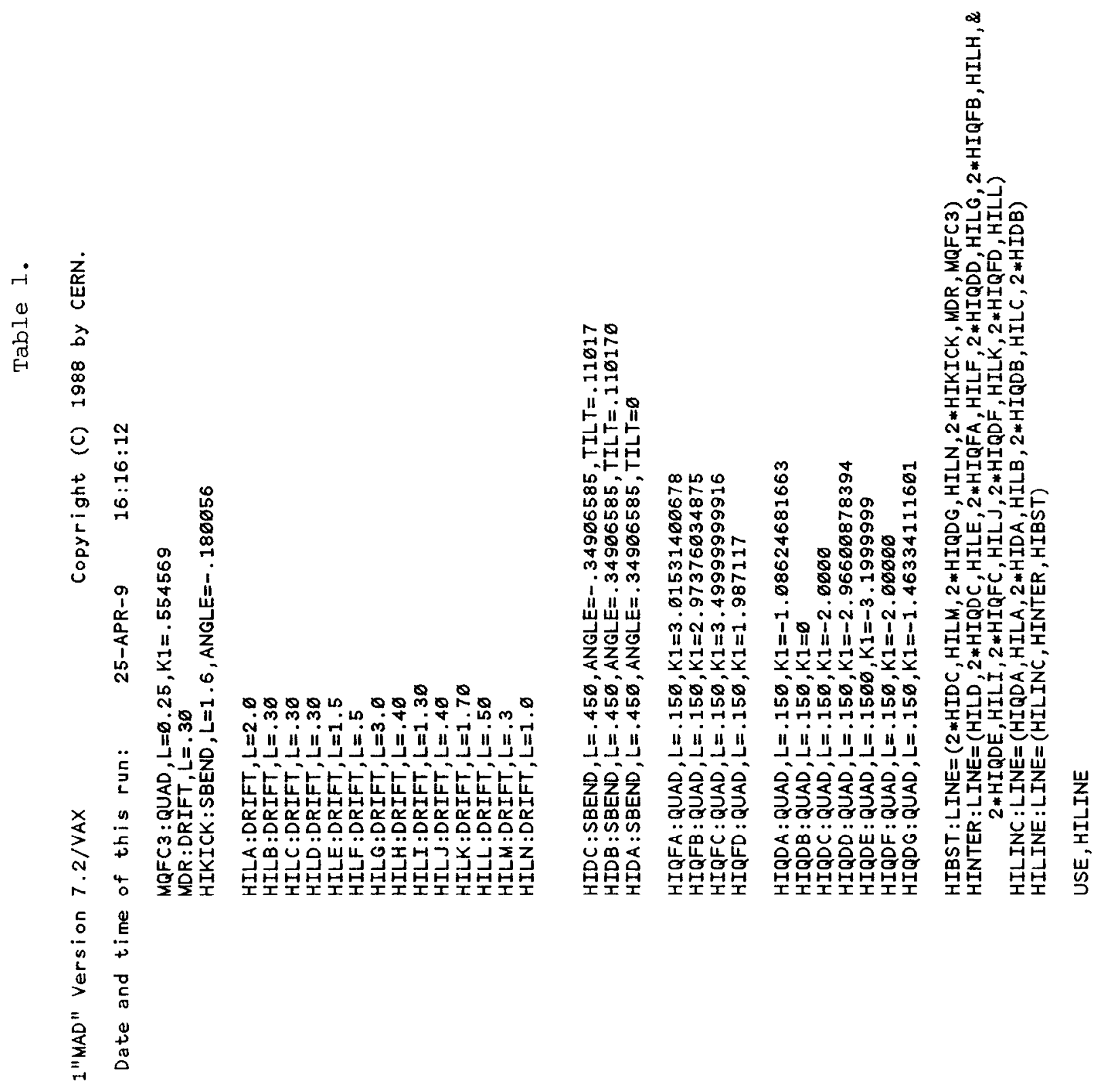




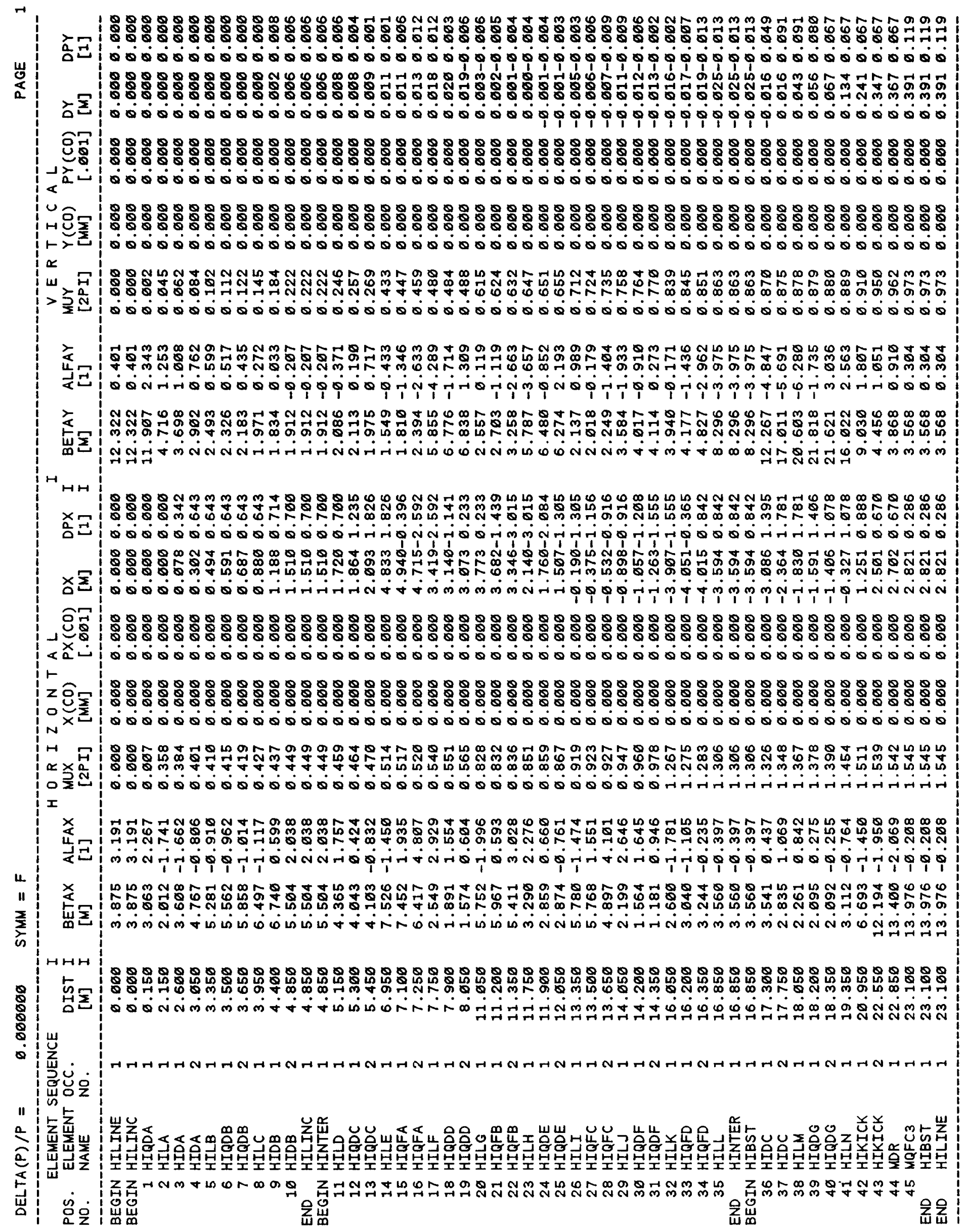




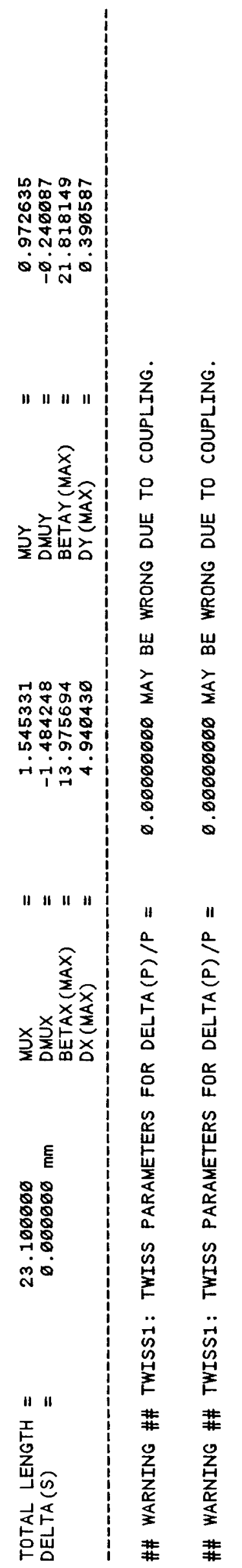


Q 00

돌

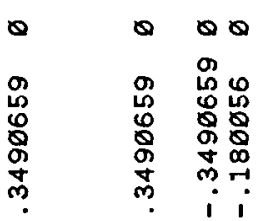

$\begin{array}{lll}10 & 10 & 10 \\ 8 & 8 & 8\end{array}$

$\dot{\theta} \pm \quad \dot{0}+\dot{0} \dot{0}$

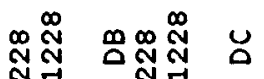

영ำ

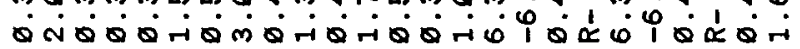
농 \%ั

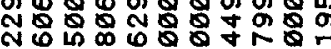
ஸैษ

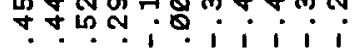

00000000000000

00000000000000 의

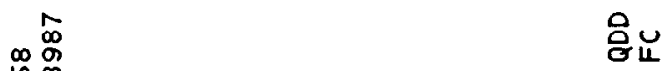

10 $\infty$

$\infty \%$

000000000000

Lu.

乙 ⿷匚

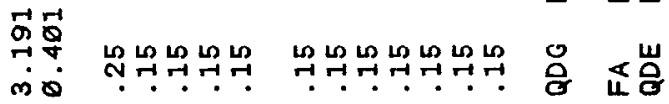

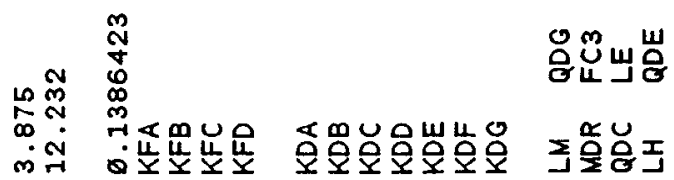

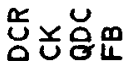

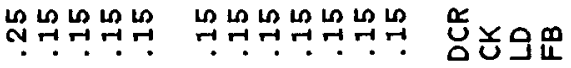

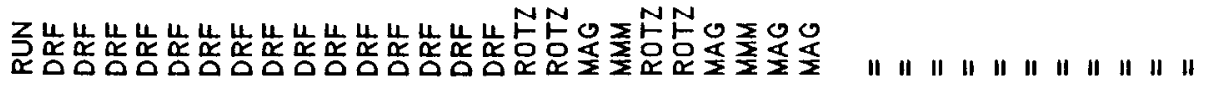

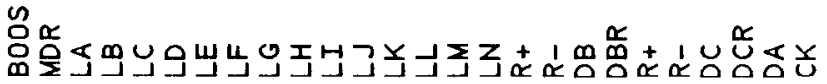

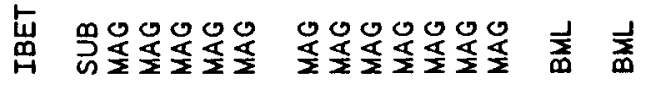

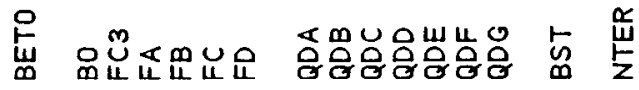




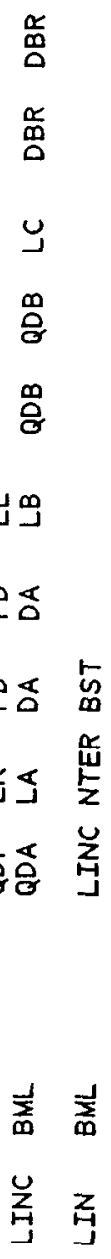




\begin{tabular}{|c|c|c|c|c|c|c|c|c|c|}
\hline \multirow{4}{*}{ 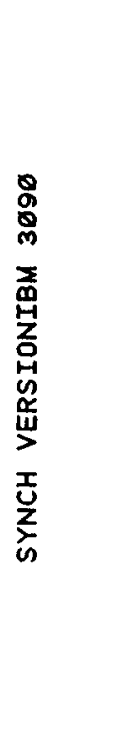 } & 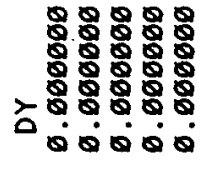 & 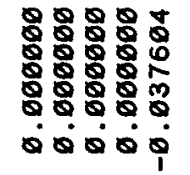 & 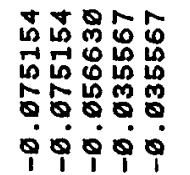 & 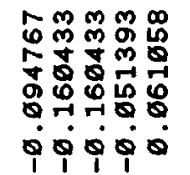 & 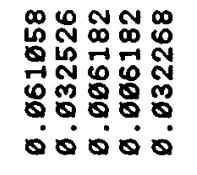 & 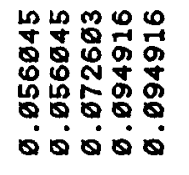 & 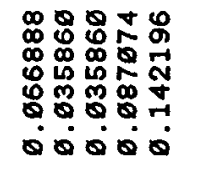 & 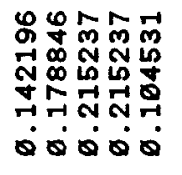 & 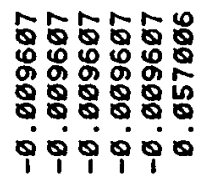 \\
\hline & 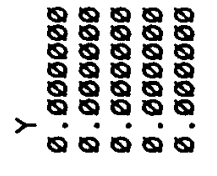 & 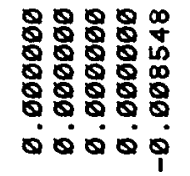 & 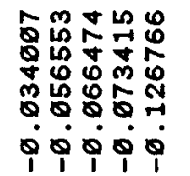 & 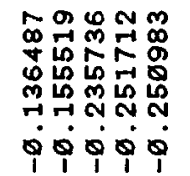 & 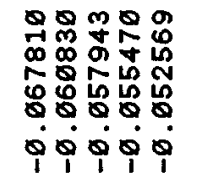 & 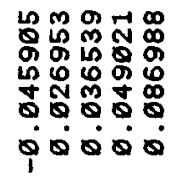 & 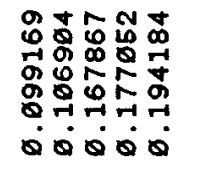 & 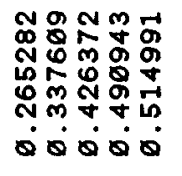 & 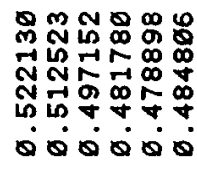 \\
\hline & 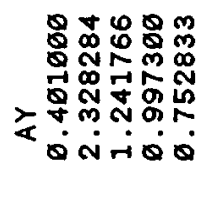 & 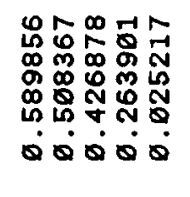 & 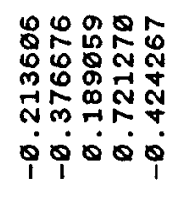 & 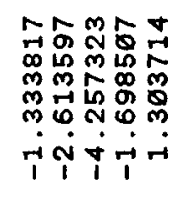 & 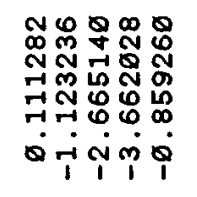 & 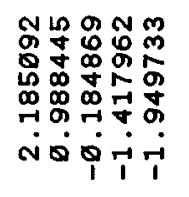 & 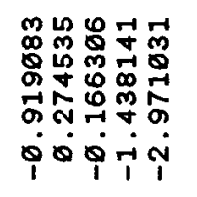 & 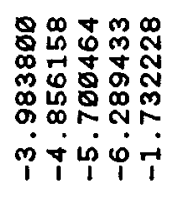 & 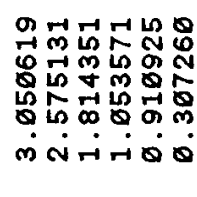 \\
\hline & 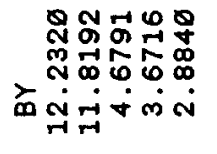 & 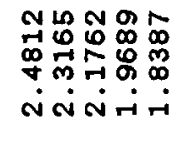 & 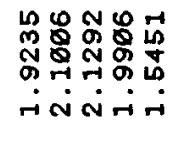 & 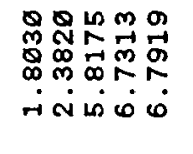 & 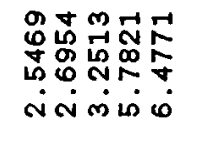 & 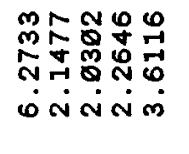 & 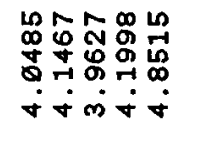 & 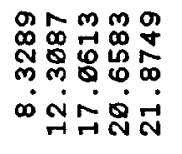 & 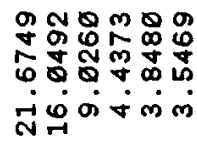 \\
\hline & 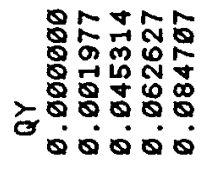 & 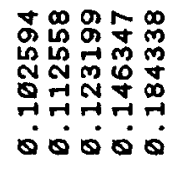 & 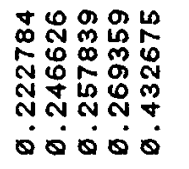 & 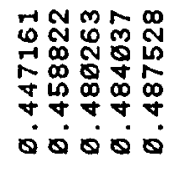 & 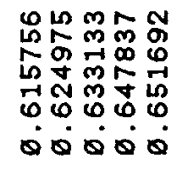 & 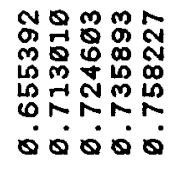 & 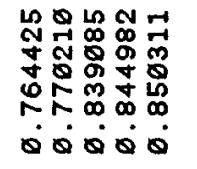 & 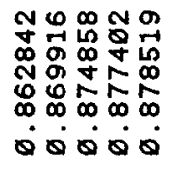 & 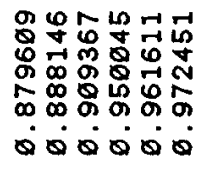 \\
\hline & 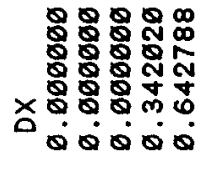 & 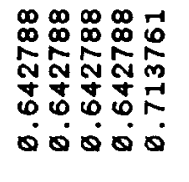 & 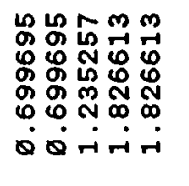 & 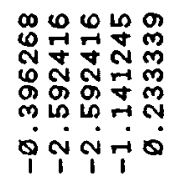 & 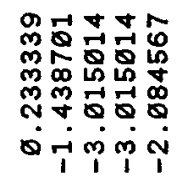 & 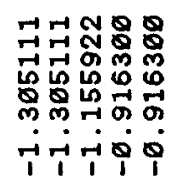 & 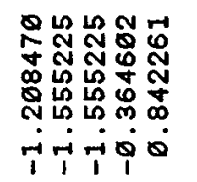 & 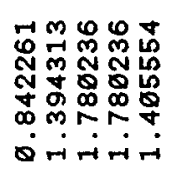 & 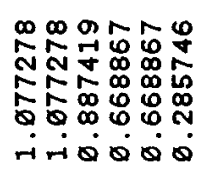 \\
\hline & 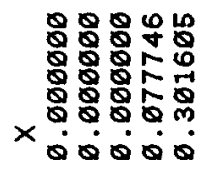 & 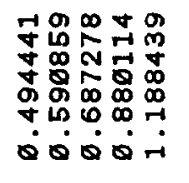 & 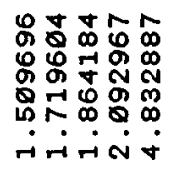 & 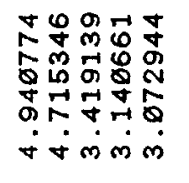 & 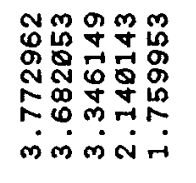 & 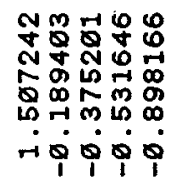 & 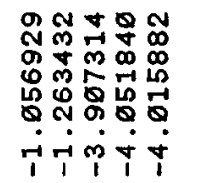 & 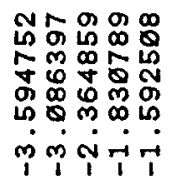 & 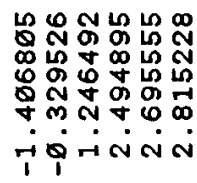 \\
\hline & 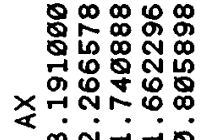 & 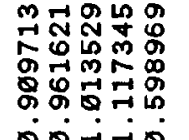 & 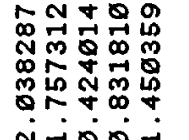 & 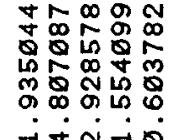 & 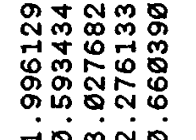 & 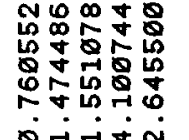 & 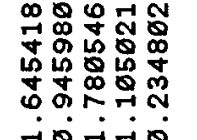 & 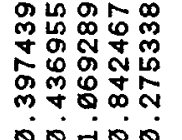 & 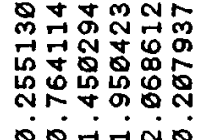 \\
\hline & & i & arti & $+\infty$ & & 17 & 1 & & 11 \\
\hline & 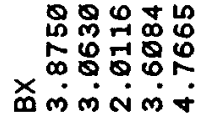 & 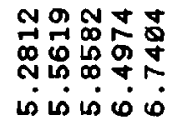 & 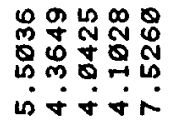 & 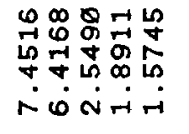 & 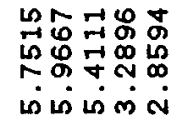 & 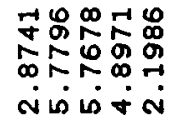 & 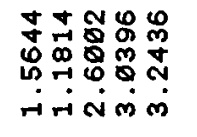 & 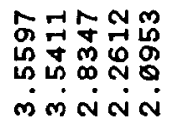 & 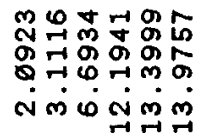 \\
\hline 吕 & 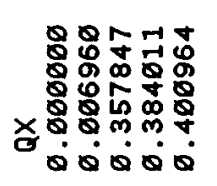 & 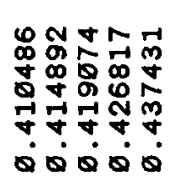 & 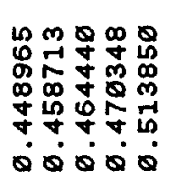 & 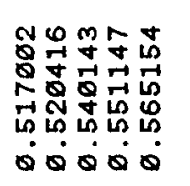 & 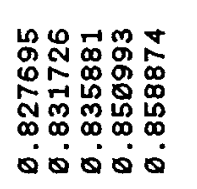 & 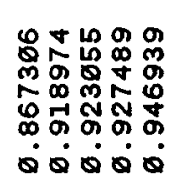 & 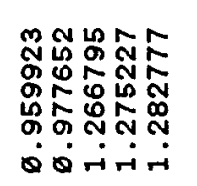 & 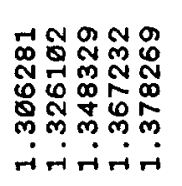 & 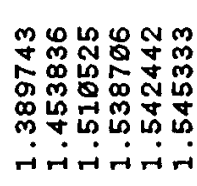 \\
\hline 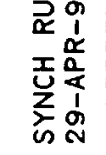 & 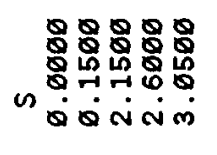 & 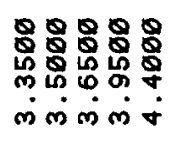 & 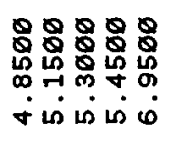 & 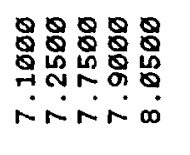 & 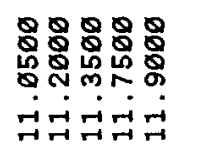 & 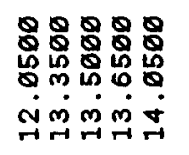 & 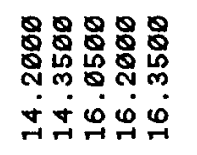 & 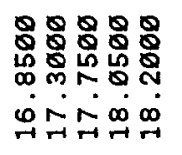 & 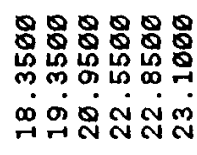 \\
\hline & 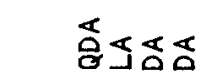 & 988 & 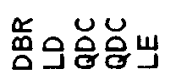 & 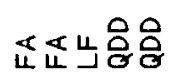 & 号思思工岂 & 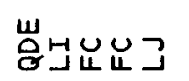 & 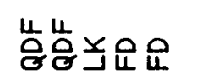 & 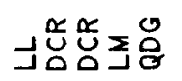 & 㞧 \\
\hline & $\cos ^{\circ}$ & $\cos \theta$ & 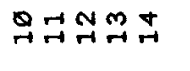 & 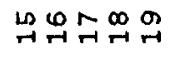 & 䞸 $N \stackrel{\sim}{N}$ & 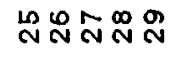 & 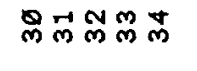 & 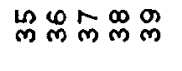 & +4 \\
\hline
\end{tabular}



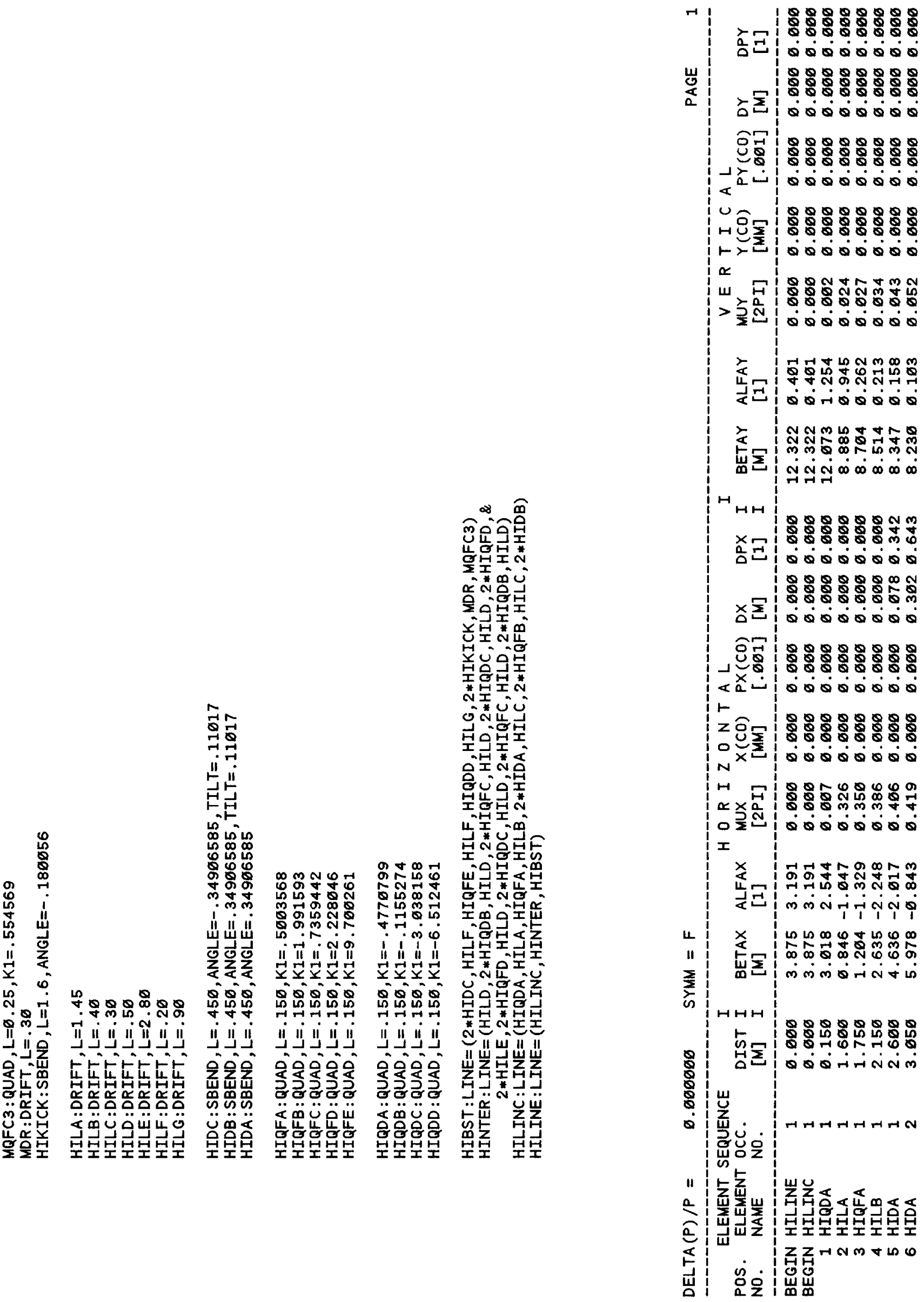


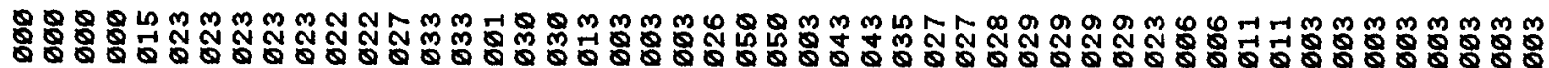
Q Q

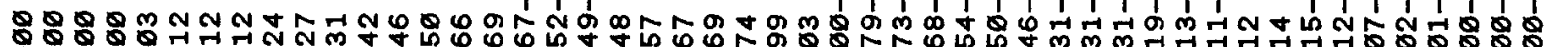

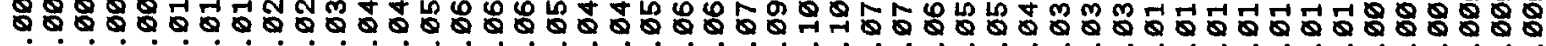

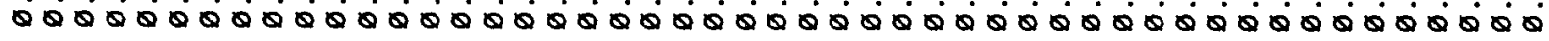
: वें :

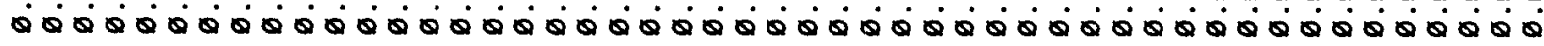

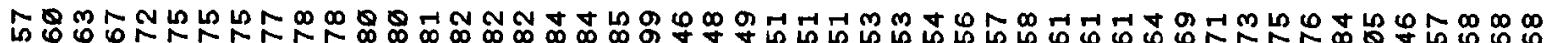

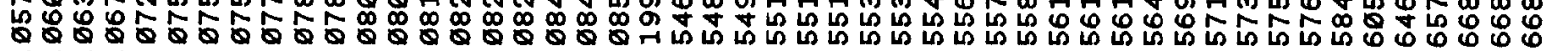

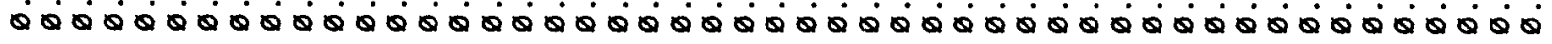

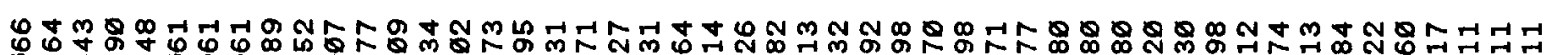

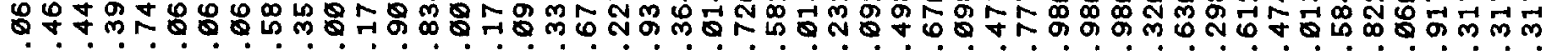
ه p p p

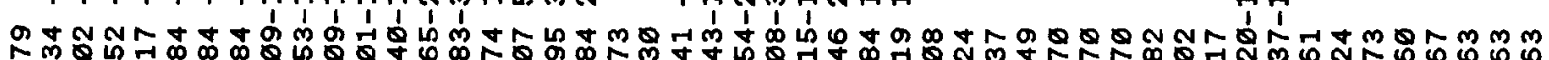

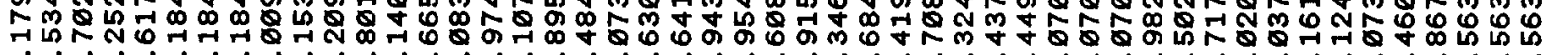

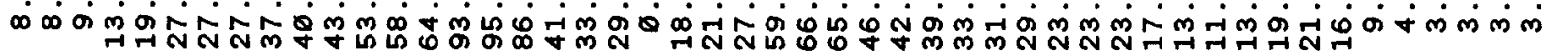

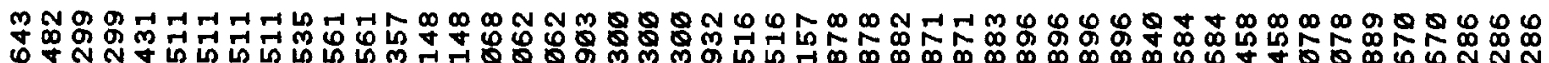

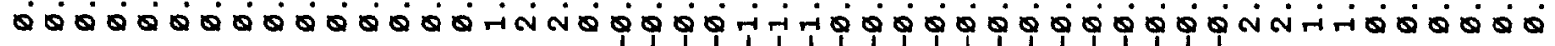

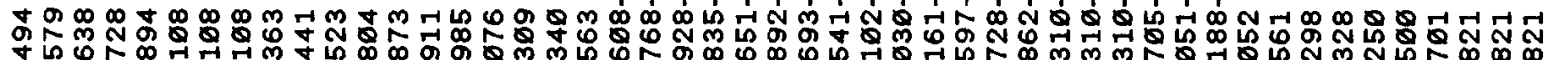

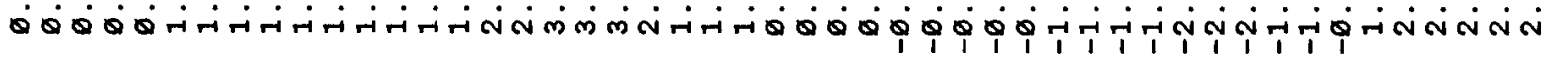

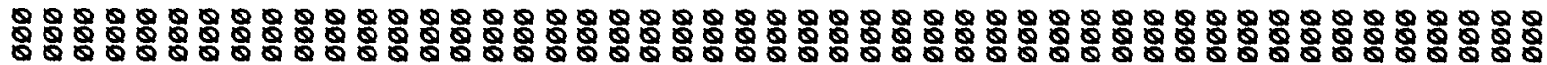

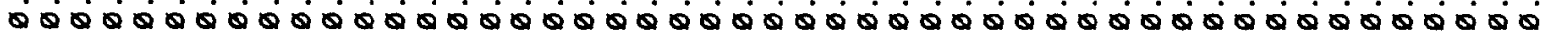

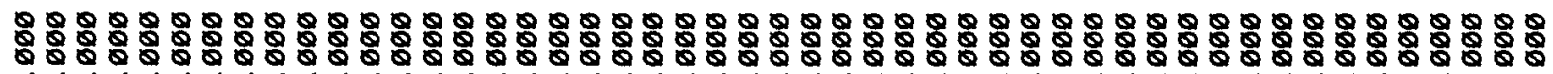

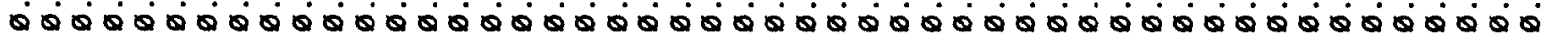

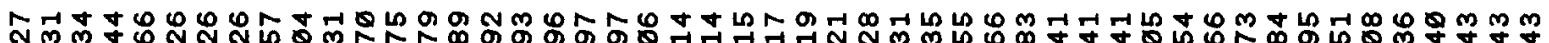
군?

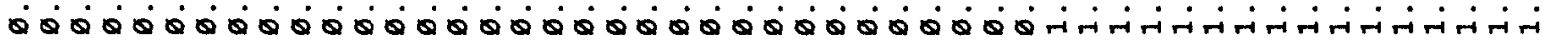

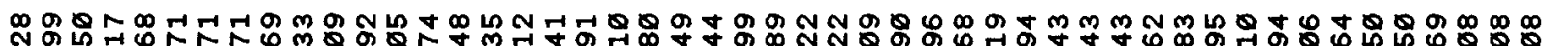

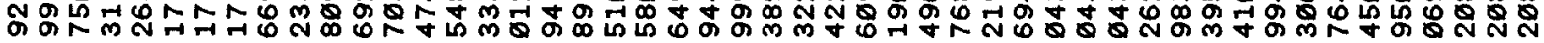

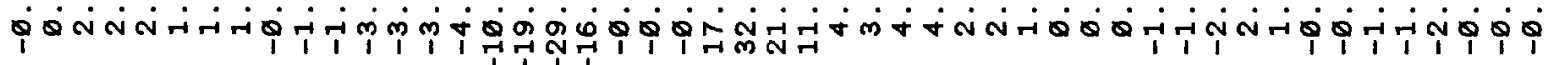

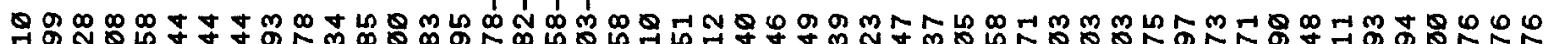

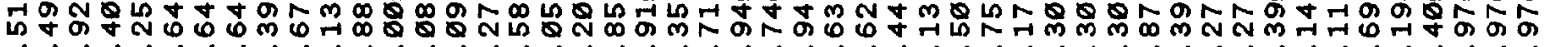

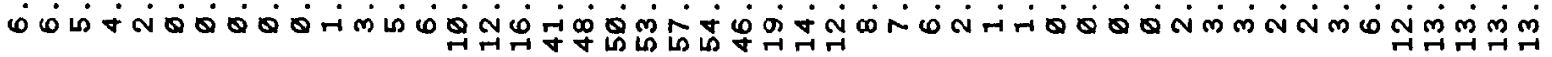

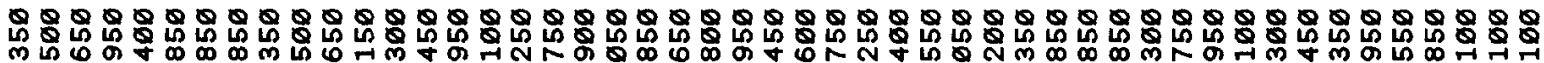
m mm

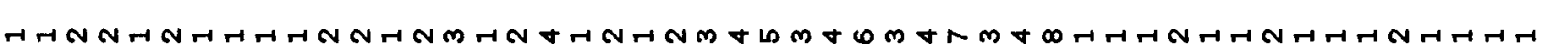

